\title{
Perfil epidemiológico de saúde bucal de salva-vidas, Salvador, Bahia, Brasil, 2012
}

\author{
Epidemiological profile of lifeguards, Salvador, Bahia, Brazil, 2012
}

Bianca de Novaes Pimenta*

Vanessa Vasconcelos Lessa*

Camila Sane Viena***

Rafael Moreira Daltro ${ }^{* * *}$

Tatiana Frederico de Almeida ${ }^{* * * *}$

Ricardo Araújo da Silva ${ }^{* * * * *}$

\section{Resumo}

As condições de saúde bucal são influenciadas por fatores de diversas origens, dentre os quais se destacam as condições ocupacionais. Objetivo: este estudo tem como objetivo a realização de um inquérito epidemiológico sobre saúde bucal de trabalhadores do Salvamar (órgão da Prefeitura Municipal de Salvador responsável pelos serviços de salva-vidas), que exercem a função de salva-vidas e que, por conseguinte, encontram-se cotidianamente expostos à radiação solar. Sujeitos e método: trata-se de um estudo descritivo, de corte transversal, conduzido com uma amostra de conveniência de salva-vidas que, voluntariamente, se submeteram ao exame bucal que incluiu a avaliação de cárie dentária, uso de prótese, alterações periodontais e lesões de mucosa oral. Os exames foram realizados por uma estudante de graduação do curso de Odontologia da Escola Bahiana de Medicina e Saúde Pública, no decorrer do segundo semestre do ano de 2012. Resultados: os dados foram analisados descritivamente, sendo observado um $C P O-D$ de 9,37 e o uso de prótese dentária, bem como presença de alguma alteração periodontal entre 22,54\% dos salva-vidas. A prevalência de lesões de mucosa oral, na ordem de $21,13 \%$, foi outro dado que despertou a atenção para a necessidade de implantação de ações preventivas dirigidas a esse grupo populacional no que diz respeito à saúde bucal. Conclusão: considerando a presença e à gravidade dos agravos bucais encontrados entre os salva-vidas e o risco a que estão expostos cotidianamente, torna-se importante o plane- jamento e implantação de programas de saúde bucal no local de trabalho, visando à promoção de saúde e prevenção de riscos.

Palavras-chave: Epidemiologia. Saúde bucal. Salva-vidas.

\section{Introdução}

A saúde de uma população sofre interferência de diversos fatores como a questão socioeconômica e cultural. Outro fator, que recebe cada vez mais importância é a questão ocupacional, uma vez que, no contexto da globalização e das inovações na economia, há um aumento das exigências dos trabalhadores, prejudicando a saúde e o rendimento ${ }^{1}$. Com isso, surge a preocupação de promover serviços de saúde ao trabalhador, dando origem à concepção de saúde do trabalhador ${ }^{2}$.

A conscientização das classes trabalhadoras quanto à necessidade de tornar obrigatória a assistência à saúde do trabalhador para prevenir doenças levou esta discussão para a área odontológica ${ }^{3}$. A importância da saúde bucal para o alcance de padrões de boa qualidade de vida é evidente ${ }^{4}$. Graduanda em Odontologia, Escola Bahiana de Medicina e Saúde Pública, Salvador, Bahia, Brasil, bolsista Fundação de Amparo à Pesquisa do Estado da Bahia Graduando em Odontologia da Escola Bahiana de Medicina e Saúde Pública, Salvador, Bahia, Brasil, bolsista do Programa de Educação Tutorial OdontoBahiana, bacharelando em Saúde Interdisciplinar da Universidade Federal da Bahia

Doutora em Saúde Coletiva, Universidade Federal da Bahia, professora adjunta de Odontologia, Escola Bahiana de Medicina e Saúde Pública, Salvador, Bahia, Brasil * Mestre em Odontologia Legal, professor assistente de Odontologia, Escola Bahiana de Medicina e Saúde Pública, Salvador, Bahia, Brasil 
Assim, a Odontologia do Trabalho, enquanto especialidade odontológica, tem como objetivo analisar, interpretar e solucionar os problemas bucais que possam acontecer com os trabalhadores durante o processo de produção e consumo de bens. As doenças bucais comprometem a saúde geral do indivíduo, repercutindo de forma negativa na qualidade de vida e afetando a sua capacidade laborativa ${ }^{3-6}$. Por esses motivos é que vários estudos afirmam a necessidade de programas odontológicos nas empresas, diminuindo o afastamento desses trabalhadores do trabalho e melhorando o processo produtivo ${ }^{7}$.

Assim como as doenças sistêmicas, as doenças bucais envolvem desde fatores ambientais, genéticos e imunológicos até fatores sociocomportamentais, a exemplo da vida social, hábitos de higiene oral e acesso à assistência odontológica. Fatores ambientais ou ocupacionais são relevantes para a saúde bucal, porque a boca, pela sua posição e múltiplas funções, é vulnerável a agressões do meio externo $0^{8-9}$.

Dentre as alterações bucais potencialmente resultantes de certas exposições ocupacionais foram listadas: cárie dentária, periodontopatias, lesões da mucosa oral, descalcificações e desgastes dentais, além de sinais e sintomas, como a pigmentação de estruturas bucais (na mucosa, gengiva e lábios), sensação de secura na boca, perda de sensibilidade e paladar, entre outros sintomas ${ }^{8}$. O trabalhador que está exposto às radiações solares é mais vulnerável às alterações hiperceratóticas da mucosa oral, que podem evoluir para neoplasias malignas, como o câncer de boca ${ }^{9,10}$. Esse tipo de câncer é o maior do grupo dentre as neoplasias malignas de cabeça e pescoço ${ }^{11}$.

Este estudo tem como objetivo realizar um inquérito epidemiológico de saúde bucal em trabalhadores expostos à radiação solar no seu cotidiano, os salva-vidas de Salvador, BA. Dessa maneira, espera-se reconhecer o real quadro epidemiológico de saúde bucal nesse grupo populacional, com fito de planejar ações de prevenção e tratamento, haja vista que, até o momento, não foram encontrados estudos dessa natureza voltados para esse grupo ocupacional, tampouco existem ações odontológicas voltadas para promoção de saúde bucal.

\section{Sujeitos e método}

Este é um estudo descritivo de corte transversal, conduzido com uma amostra de conveniência de salva-vidas do Salvamar (órgão da Prefeitura Municipal de Salvador responsável pelos serviços de salva-vidas) que, voluntariamente, foram submetidos ao exame bucal de natureza epidemiológica, a fim de identificar problemas bucais.

Os exames bucais foram realizados no segundo semestre de 2012, por uma discente de Odontologia do $9^{\circ}$ semestre da Escola Bahiana de Medicina e Saúde Pública (EBMSP), devidamente treinada e calibrada, segundo critérios do último levantamento nacional de saúde bucal (Saúde Bucal Brasil $2010)^{21}$, tendo sido atingidos índices de concordância acima de $85 \%$ para todos os agravos pesquisados. Para a coleta de dados, foram utilizadas fichas que perquiriam as seguintes informações: sexo, idade, estado civil, escolaridade, renda, naturalidade, CPO-D, uso de prótese dentária, estado da prótese, sangramento gengival (referido pelo trabalhador quando presente, se espontâneo ou provocado ao escovar), alteração periodontal (exame visual da presença de recessões, abscessos e cálculo) e alteração de tecido mole. Em caso de presença de lesão de mucosa oral, foram descritos: coloração, localização, base da lesão, textura da lesão, superfície de lesão, consistência, presença de sangramento, sintomatologia dolorosa, caráter redicivantes, fator desencadeante e tempo de evolução.

Os dados foram digitados em planilha do programa Microsoft Excel e analisados descritivamente no Stata 10, no qual foram feitas análises estatísticas descritivas de frequência simples para variáveis categóricas e medidas de tendência central para variáveis contínuas.

Os salva-vidas participantes autorizaram o exame mediante assinatura do termo de consentimento livre e esclarecido. Durante o exame bucal não houve nenhum tipo de risco físico, biológico, psicológico ou moral para os participantes. Este estudo foi submetido e aprovado pelo Comitê de Ética em Pesquisa da EBMSP, em 13 de novembro de 2012, com número de CAAE 05961312.2.0000.5544.

\section{Resultados}

O estudo abrangeu uma amostra populacional de 71 trabalhadores do Salvamar, todos ocupantes do cargo de salva-vidas, que foram submetidos a exames bucais. A Tabela 1 descreve algumas características demográficas e socioeconômicas da população de estudo. 
Tabela 1 - Distribuição absoluta e percentual dos trabalhadores do Salvamar segundo suas características socioeconômicas, Salvador,BA, 2012

\begin{tabular}{|c|c|c|}
\hline & & \\
\hline & $\mathrm{n}$ & $\%$ \\
\hline \multicolumn{3}{|l|}{ Sexo } \\
\hline Masculino & 70 & $98,58 \%$ \\
\hline Feminino & 01 & $1,42 \%$ \\
\hline \multicolumn{3}{|l|}{ Escolaridade } \\
\hline Ensino fundamental incompleto & 03 & $4,22 \%$ \\
\hline Ensino fundamental completo & 09 & $12,67 \%$ \\
\hline Ensino médio incompleto & 06 & $8,45 \%$ \\
\hline Ensino médio completo & 31 & $43,66 \%$ \\
\hline Ensino superior & 22 & $30,98 \%$ \\
\hline \multicolumn{3}{|l|}{ Estado Civil } \\
\hline Solteiro & 31 & $43,66 \%$ \\
\hline Casado & 29 & $40,84 \%$ \\
\hline Viúvo & 01 & $1,40 \%$ \\
\hline Separado & 05 & $7,04 \%$ \\
\hline Relação estável & 05 & $7,04 \%$ \\
\hline \multicolumn{3}{|l|}{ Ocupação } \\
\hline Salva-vidas & 66 & $92,95 \%$ \\
\hline Administração & 03 & $4,22 \%$ \\
\hline Outros & 02 & $2,81 \%$ \\
\hline \multicolumn{3}{|l|}{ Renda } \\
\hline Até 01 SM & 02 & $2,81 \%$ \\
\hline Entre 01 e 03 sm & 24 & $33,80 \%$ \\
\hline Entre 03 e 05 sm & 34 & $47,88 \%$ \\
\hline Maior que 05 sm & 04 & $5,63 \%$ \\
\hline Não se aplica & 01 & $1,40 \%$ \\
\hline Sem informação & 06 & $8,45 \%$ \\
\hline \multicolumn{3}{|l|}{ Naturalidade } \\
\hline Salvador & 46 & $64,78 \%$ \\
\hline Interior da Bahia & 08 & $11,12 \%$ \\
\hline Outro estado & 03 & $4,22 \%$ \\
\hline Sem informação & 14 & $19,71 \%$ \\
\hline
\end{tabular}

Em relação ao gênero, o grupo era formado por $98,59 \%$ (70) de indivíduos do sexo masculino e $1,41 \%$ (1) do sexo feminino. Quanto ao estado civil, verificou-se que $43,66 \%$ dos salva-vidas eram solteiros e $40,85 \%$ casados. Os demais se enquadraram entre viúvos, separados e conviventes (em união estável). Em relação à naturalidade, pôde-se observar que $64,79 \%$ (46) dos trabalhadores eram naturais de Salvador, BA e 11,27\% (11) do interior da Bahia.

Quanto à escolaridade, a maioria dos trabalhadores indicou ter cursado o ensino médio, $43,66 \%$, e $30,99 \%$ o ensino superior. A renda auferida pela maioria dos trabalhadores encontrava-se dentro da faixa de três a cinco salários mínimos $(47,89 \%)$, seguida pela faixa entre um e três salários mínimos, $33,80 \%$, e daqueles que recebiam mais do que cinco salários mínimos, 5,63\%. Um percentual de $2,82 \%$ (2) trabalhadores auferiam até o valor de um salário mínimo. A principal ocupação dos trabalhadores era de salva-vidas, $92,96 \%$, seguida da área administrativa, com $4,23 \%$ e, por $2,82 \%$ de outros cargos.

Os resultados obtidos, relacionados ao exame clínico, apontaram um índice CPO-D com valor médio igual a 9,37. Conforme é possível observar na Figura 1, em relação à experiência de cárie, os dados analisados revelaram um percentual de 2,86\% de dentes cariados, $20,15 \%$ de dentes perdidos, e $76,99 \%$ de dentes obturados.

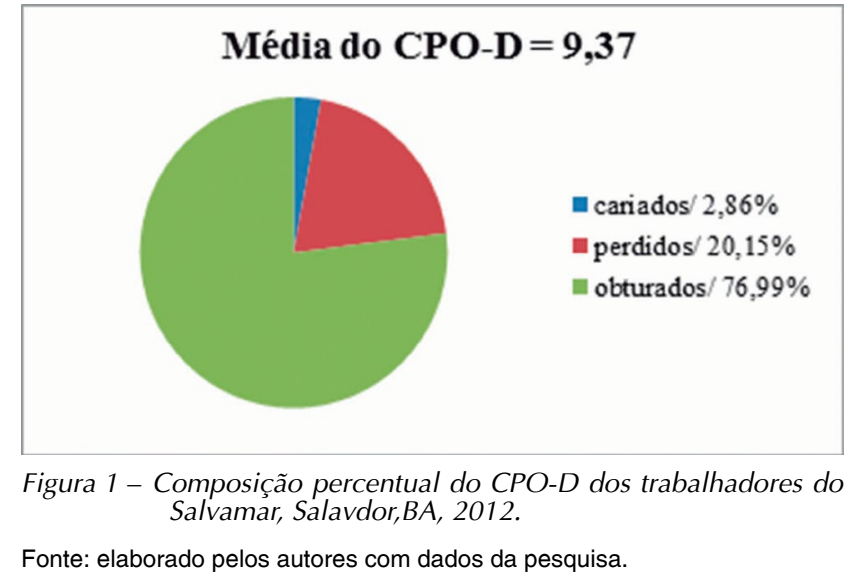

Sobre as demais condições de saúde bucal avaliadas, a Tabela 2 apresenta informações quanto ao uso e ao estado das próteses. Verificou-se a utilização de prótese, de qualquer tipo, em $22,54 \%$ dos trabalhadores (16), sendo que 18,75\% (3) das próteses estavam em bom estado.

Tabela 2 - Distribuição absoluta e percentual dos trabalhadores do Salvamar em relação ao uso e estado das próteses dentárias, Salvador-BA, 2012

\begin{tabular}{|c|c|c|}
\hline & \multirow[b]{2}{*}{$\mathrm{N}$} & \multirow[b]{2}{*}{$\%$} \\
\hline & & \\
\hline \multicolumn{3}{|c|}{ Faz uso de prótese } \\
\hline Não & 55 & $77,46 \%$ \\
\hline Sim & 16 & $22,53 \%$ \\
\hline \multicolumn{3}{|c|}{ Estado da prótese } \\
\hline Bom & 03 & $18,75 \%$ \\
\hline Ruim & 13 & $81,25 \%$ \\
\hline
\end{tabular}

Fonte: elaborado pelos autores com dados da pesquisa. 
A Tabela 3 descreve os resultados de alterações periodontais e sangramento gengival. Entre os 71 trabalhadores examinados, $22,54 \%$ dos indivíduos (16) apresentaram algum tipo de alteração periodontal (bolsa, abscessos, recessões, cálculo). Nos demais trabalhadores $77,46 \%$, não se verificou presença de alterações periodontais. Quanto à presença de sangramento gengival, pôde-se observar que cerca de $30 \%$ (23) dos trabalhadores não fizeram referência a nenhum tipo, $60 \%$ (43) tinham sangramento ao escovar os dentes, e outros 7\% (5) sangravam espontaneamente.
Tabela 3 -Distribuição absoluta e percentual dos trabalhadores do Salvamar quanto à alteração periodontal e a sangramento gengival, Salvador, BA, 2012

\begin{tabular}{l|r|r}
\cline { 2 - 3 } & $\mathrm{N}$ & $\%$ \\
\hline Alteração periodontal & 55 & $77,46 \%$ \\
Não & 16 & $22,53 \%$ \\
Sim & & \\
Sangramento gengival & 23 & $32,39 \%$ \\
Não sangra & 43 & $60,56 \%$ \\
Sangra ao escovar & 05 & $7,04 \%$ \\
Sangramento espontâneo &
\end{tabular}

Fonte: elaborado pelos autores com dados da pesquisa.

A Tabela 4 fornece dados referentes às alterações de mucosa, que foram constatadas entre $21,13 \%$ (15) da população de estudo.

Tabela 4 - Distribuição absoluta e percentual dos trabalhadores do Salvamar segundo as alterações de mucosa, Salvador,BA, 2012

\begin{tabular}{|c|c|c|c|c|c|}
\hline Característica das lesões & $\mathrm{N}$ & $\%$ & Base das lesões & $\mathrm{N}$ & $\%$ \\
\hline Úlcera & 3 & $20,00 \%$ & Pediculada & 1 & $6,66 \%$ \\
\hline Nódulo & 2 & $13,33 \%$ & Séssil & 6 & $40,00 \%$ \\
\hline Mancha & 4 & $26,66 \%$ & Não se aplica & 8 & $53,33 \%$ \\
\hline Pápula & 1 & $6,66 \%$ & Textura das lesões & & \\
\hline Bolha & 1 & $6,66 \%$ & Lisa & 11 & \\
\hline Vesícula & 1 & $6,66 \%$ & Rugosa & 4 & $26,66 \%$ \\
\hline Outro & 2 & $13,33 \%$ & Superfície das lesões & & \\
\hline Não se aplica & 1 & $6,66 \%$ & Brilhante & 9 & $60,00 \%$ \\
\hline Coloração das lesões & & & Opaca & 5 & $33,33 \%$ \\
\hline Branca & 2 & $13,33 \%$ & Não se aplica & 1 & $6,66 \%$ \\
\hline Vermelha & 8 & $53,33 \%$ & Sintomatologia dolorosa & & \\
\hline Amarronzada & 4 & $26,66 \%$ & Sim & 1 & $6,66 \%$ \\
\hline Acinzentada & 1 & $6,66 \%$ & Não & 14 & $93,33 \%$ \\
\hline Localização das lesões & & & Fator desencadeante das lesões & & \\
\hline Mucosa jugal & 6 & $40,00 \%$ & Trauma & 6 & $40,00 \%$ \\
\hline Assoalho de boca & 1 & $6,66 \%$ & Outros & 3 & $20,00 \%$ \\
\hline Língua & 2 & $13,33 \%$ & Impossível determinar & 4 & $26,66 \%$ \\
\hline Rebordo alveolar & 1 & $6,66 \%$ & Não se aplica & 2 & $13,33 \%$ \\
\hline Gengiva & 1 & $6,66 \%$ & & & \\
\hline Lábios & 4 & $26,66 \%$ & & & \\
\hline
\end{tabular}

Fonte: elaborado pelos autores com dados da pesquisa.

Foi possível observar que as principais características das alterações de mucosa encontradas foram: $26,67 \%$ (4) manchas, $20 \%$ (3) úlceras e $13,33 \%$ (2) nódulos. Quanto à cor dessas lesões, $53,33 \%$ tinham coloração avermelhada e $26,67 \%$ eram de cor amarronzada.

Sobre a localização das lesões de mucosa oral, a maioria tinha sítio na mucosa jugal, com $40 \%$ (6), seguida das localizadas em lábio, com $26,67 \%$ (4), e língua, com $13,33 \%$ (2). Dessas lesões, $53,33 \%$ (8) não possuíam base, seguidas de lesões que possuíam base séssil, com $40 \%$ (6), e de lesões com base pediculada, com $6,67 \%$ (1). Cerca de $70 \%$ das lesões
(11) apresentaram uma textura lisa, e 26,67\% (4) apresentaram textura rugosa. Cerca de $60 \%$ (9) de lesões tinham superfície brilhante, seguidas de lesões opacas, com $33,33 \%$ (5).

A maioria das lesões não sangrava, tendo uma sintomatologia não dolorosa, representando 93,33\% (14) dos casos, e com caráter não redicivante. $\mathrm{O}$ principal fator desencadeante dessas lesões foi o trauma $40 \%$, seguido de fatores que foram impossíveis de identificar, $26,67 \%$, e de outros fatores $20 \%$. 


\section{Discussão}

Este estudo demonstrou a presença e gravidade de lesões bucais importantes entre os salva-vidas de Salvador,BA. Em relação à cárie dentária, o CPO-D encontrado, de 9,37, foi menor quando comparado ao de trabalhadores de outras ocupações, como de trabalhadores de uma cooperativa da unidade de Maravilha, estado de Santa Catarina, em que o CPO-D foi de $20,2^{12}$, e de trabalhadores de uma padaria e confeitaria de Blumenau-SC, com CPO-D de $20,95^{13}$. Esses últimos estão mais expostos a alimentos cariogênicos durante o processo de trabalho, e sabe-se que a dieta exerce um papel fundamental no desenvolvimento da cárie, pela fermentação de carboidratos.

No Brasil, segundo resultados do último levantamento nacional, os adultos na faixa etária de 35 a 44 anos tinham o CPO-D de $16,75^{14}$, já em um estudo realizado para avaliar as condições de saúde bucal de crianças, adolescentes e adultos no município de Salvador, pôde-se observar que na faixa etária dos 35 aos 44 anos, os indivíduos apresentaram o CPO-D de $14,1^{15}$, mostrando ter um CPO-D maior que o dos trabalhadores salva-vidas. Fatores socioeconômicos, comportamentais e culturais também podem influenciar no hábito de higiene bucal, no uso de fluoretos, no baixo consumo de açúcar, no autocuidado com a saúde bucal, o que também influencia o aparecimento da doença ${ }^{12}$.

Entre os salva-vidas, $72,46 \%$ não faziam uso de nenhum tipo de prótese dentária e dentre aqueles que faziam uso, $18,75 \%$ das próteses estavam em bom estado. Um estudo realizado com trabalhadores atendidos em um Núcleo de Saúde Ocupacional de Campina Grande, -PB relatou que 36\% dos examinados faziam uso de algum tipo de prótese, e que $80,1 \%$ necessitavam de prótese ou trocar a que já utilizavam ${ }^{16}$. O SB Brasil 2010 (último levantamento de saúde bucal nacional) verificou que $67,2 \%$ dos indivíduos entre 35 a 44 anos não usavam ou necessitava do uso de prótese dentária ${ }^{14}$, já em Salvador, $67 \%$ dos indivíduos nessa mesma faixa etária também não utilizavam prótese ${ }^{15}$.

O uso de prótese dentária pode causar alterações na mucosa bucal, podendo gerar lesões resultantes da sua má adaptação ou da higienização inadequa$\mathrm{da}^{17}$. A necessidade de reabilitação bucal está relacionada a populações com uma faixa etária superior a quarenta anos e a fatores socioeconômicos, dentre eles, a falta de acesso a tratamentos odontológicos. Mesmo sendo uma alternativa mais prática e econômica, existe uma oferta insuficiente de reabilitação protética no país, sendo quase inexistente em serviços públicos ${ }^{18}$. A oferta de reabilitação protética à população proporciona uma melhor qualidade de vida, por melhorar a alimentação, a fonação e a estética $^{16,18-20}$.
Nesse estudo, $77,46 \%$ dos trabalhadores não apresentavam alterações periodontais, ligadas a bolsas, abscessos, cálculos, dentre outras variações. Os estudos mostram que a presença e gravidade das doenças periodontais nas populações são frequentes. Os resultados do SB Brasil de 2003 mostraram uma prevalência de $9,9 \%$ bolsas de $4 \mathrm{~mm}$ ou mais em indivíduos na faixa etária de 35 a 44 anos de idade $^{21}$. Já os resultados do SB Brasil de 2010 mostraram que os indivíduos nessa mesma faixa etária possuíam uma prevalência de $28,6 \%$ de cálculo e $19,4 \%$ de bolsas periodontais ${ }^{15}$.

Quanto à presença de sangramento gengival, cerca de $70 \%$ dos salva-vidas apresentaram essa alteração espontaneamente e ao escovar os dentes. Isso se dá pelo acúmulo de alimentos, que infecciona a gengiva, e pela má higienização ${ }^{22}$.

A mucosa oral está sujeita a várias alterações, que se manifestam de várias formas. $\mathrm{O}$ indivíduo exposto a agentes ocupacionais (mecânicos, físicos e químicos) pode desencadear alterações do tecido mole, que aparecem sob diferentes aspectos clíni$\cos$ (formas, cores, consistência, textura, entre outras características) $)^{3,4,23}$. Observou-se que 21,13\% dos salva-vidas apresentaram alteração de mucosa, sendo esse percentual maior quando comparado com outros estudos. Uma investigação em trabalhadores de uma indústria metalúrgica da região metropolitana de Salvador observou que a grande maioria dos trabalhadores, de $70,6 \%$ a $90,7 \%$, não possuía nenhum tipo de alteração de mucosa bucal, e, ainda, que a maioria das lesões era de cor vermelha, maior frequência na região do palato, seguida do lábio e do rebordo alveolar ${ }^{24}$.

Esses achados contrapõem-se aos dados obtidos neste estudo, pois constatou-se que a maioria das lesões apresentadas nos salva-vidas era avermelhada, seguida de lesões amarronzadas, que se localizavam com maior frequência na região da mucosa jugal, seguida dos lábios e língua.

Sabe-se que, de acordo com a sua área de atuação, o trabalhador está exposto a agentes de riscos, que podem ser classificados como agentes químicos (ácidos, metais, açúcar, entre outros), agentes físi$\cos$ (altas e baixas temperaturas, exposição à radiação, entre outros) e agentes mecânicos (pregos, fios de costura, lápis, grampos, entre outros $)^{4}$. Tais agentes devem ser considerados durante 0 atendimento odontológico, pois estão relacionados com o aparecimento de diversos problemas bucais ${ }^{6,25}$. A boca apresenta diversas funções, como mastigação, fonação e deglutição, e devido a essa multifuncionalidade, bem como sua localização no corpo, se torna vulnerável a diversas agressões externas ${ }^{26}$. Essas alterações podem ser, entre outras, herpes, doença periodontal, lesões de cárie, como também lesões dos tecidos moles pela exposição excessiva ao sol, já que a radiação sobre os lábios pode causar queilite actínica e câncer ${ }^{3}$. 
Dentre os achados na literatura, a grande maioria das lesões bucais encontradas nos indivíduos que trabalham sob exposição solar eram as lesões pré-cancerizáveis, em especial a queilite actínica, que é uma lesão labial. Os lábios são muito suscetíveis à radiação, visto que possuem uma pequena camada de epitélio, menor proteção de melanina, além da baixa secreção de glândulas ${ }^{27}$.

O câncer de lábio pode ser classificado como um dos mais associados à exposição solar relacionada à condição de trabalho sem proteção ${ }^{10}$. Podendo ser diagnosticado pelo cirurgião dentista, por meio do aparecimento de uma pequena lesão (queilite actínica) que, com o passar do tempo, evolui para uma lesão maligna ${ }^{28}$.

Um estudo realizado em Natal,-RN, mostrou o quanto os trabalhadores de praia estão expostos às radiações UV por conta da sua profissão, e como isso está intimamente ligado ao surgimento da queilite actínica $^{27}$. Nesse caso, medidas preventivas podem ser adotadas, como programas de saúde bucal que incentivem e orientem o uso de protetores labiais em trabalhadores que precisam ficar expostos ao ar livre $^{10}$, a exemplo dos salva-vidas, expostos a altas e baixas temperaturas durante os dias de trabalho, assim como à radiação solar.

Foi possível observar que a grande maioria dos trabalhadores participantes na amostra recebia menos de cinco salários mínimos, ou seja, não tinham alto nível socioeconômico. Sabe-se que quanto menor o nível socioeconômico dos indivíduos, maior será o risco de essa população contrair o câncer bucal ${ }^{29}$.

Inúmeras justificativas podem ser elencadas para a implantação de serviços odontológicos em locais de trabalho, como a alta prevalência de lesões de cárie e alterações periodontais em determinadas ocupações, a detecção precoce de alterações de tecido mole e câncer de boca, a melhoria do estilo de vida e do rendimento em locais de trabalho ${ }^{1-4}$. Para a proteção dos profissionais que trabalham ao ar livre, foi promulgada a Lei 552/2009, que limita as horas de trabalho, estabelece intervalos para descanso, e promove remuneração extra para os indivíduos que trabalham expostos ao sol' ${ }^{28}$. Entretanto, sabe-se que os trabalhadores em geral não têm tanta informação quanto ao devido uso de proteção solar ${ }^{30}$.

As condições de saúde geral estão, muitas vezes, relacionadas às condições de saúde bucal e psicológica. Em virtude disso, sabe-se que qualquer alteração nesse tripé irá causar desconforto e desequilíbrio para o indivíduo, prejudicando suas relações interpessoais, qualidade de vida e produção ocupacional ${ }^{5}$. Além disso, já está comprovado que a instituição de programas voltados para a promoção de saúde no local de trabalho é fundamental para o controle de diversas alterações da cavidade bucal, bem como na diminuição de idas do trabalhador aos serviços de saúde bucal ${ }^{7}$.

Entre os limites desse estudo, deve-se salientar o uso de uma amostra de conveniência, o desenho de estudo de corte transversal e o seu caráter descritivo. No entanto, o presente estudo revelou o quadro epidemiológico de saúde bucal de um grupo ocupacional exposto principalmente à radiação solar, cujos malefícios à saúde já estão demonstrados na literatura. Além disso, ressalta-se que até o presente momento não foram encontradas investigações epidemiológicas voltadas para esses trabalhadores.

\section{Conclusão}

Os resultados deste estudo revelaram que os salva-vidas de Salvador apresentam níveis de cárie, doença periodontal e uso de prótese dentária menores quando comparados aos resultados obtidos pela literatura nacional e internacional. Entretanto, foi elevada a prevalência de lesões de mucosa, provavelmente pela exposição solar a que estão submetidos cotidianamente por conta da sua ocupação.

Dessa forma, considerando a presença e a gravidade dos agravos bucais encontrados entre os salva-vidas, o risco a que estão expostos cotidianamente e a influência que tais alterações provocam em sua qualidade de vida e ocupacional, torna-se importante o planejamento e implantação de programas de saúde bucal no local de trabalho, visando à promoção de saúde e prevenção de riscos. Dessa forma, tanto o trabalhador quanto o empregador serão beneficiados.

\section{Abstract}

Oral health conditions are influenced by factors of various sources, among which occupational conditions stand out. Objective: This study aimed to perform an epidemiological oral health survey with employees at Salvamar (agency of the Salvador City Hall, responsible for lifeguard services), who are lifeguards, and therefore, exposed to solar radiation in their daily lives. Subjects and method: It is a cross-sectional, descriptive stu$d y$, conducted with a convenience sample of lifeguards, who voluntarily underwent oral examination, which included the assessment of dental caries, use of prosthesis, periodontal changes, and oral mucosa injuries. The exams were performed by an undergraduate dentistry student from the Medicine and Public Health School of the state of Bahia, Brazil, throughout the second school period of 2012. Results: Data were descriptively analyzed, and a DMFT index (decayed, missing, and filled teeth) of 9.37 was observed, 22.54\% of lifeguards used dental prosthesis and presented some kind of periodontal change. The prevalence of oral mucosa injuries of $21.13 \%$ was a piece of data that drew attention to the need for applying preventive actions for this population group regarding oral health. Conclusion: Considering the presence and severity of dental problems found among the lifeguards, and the risks they are daily exposed to, it is important to plan and implement oral health programs in the workplace, aiming for health promotion and risk prevention.

Keywords: Epidemiology. Oral health. Lifeguards. 


\section{Referências}

1. Pizzato E, Garbin, CAS. Odontologia do trabalho: implantação da atenção em saúde bucal do trabalhador. Odontologia Clin Cientif 2006; 5(2):99-102.

2. Almeida T, Vianna M. O papel da epidemiologia no planejamento nas ações de saúde bucal do trabalhador. Saúde Soc $2005 ; 14(3): 144-54$.

3. Peres SHCS, Theodoro DS, Ribeiro DA, Ávila ED, Greghi GA, Silva RPR. Odontologia do trabalho: doenças e lesões na prática profissional. Rev Odontol Araçatuba 2006; 27(1):54-8.

4. Teles MP, Almeida TF, Cangussu, MCT, Vianna MIP. Exposição ocupacional e saúde bucal dos trabalhadores. Rev Cienc Med Biol 2006; 5(1):48-54.

5. Guerra MJC, Greco RM, Leite ICG, Ferreira EF, Paula, MVQ. Impact of oral health conditions on the quality of life of workers. Cien Saude Colet [periódico on-line] 2014 [citado 2014 dez. 8]; 19(12):4777-86. Disponível em: url:http://www.scielo. bt/scielo.php?pid=S1413-81232014001204777\&script $=$ sci arttex\&tlng=pt.

6. Xavier AF. Doenças e manifestações orais relacionadas ao trabalho. [Monografia de Especialização em Odontologia do Trabalho]. Contagem: Instituto de Ciências da Saúde Funorte/Soebras; 2012.

7. Ide R, Mizoue T, Tsukiyama Y, Ikeda M, Yoshimura T. Evaluation of oral health promotion in the workplace: the effects on dental care costs and frequency of dental visits. Community Dent Oral Epidemiol 2001; 29:213-9.

8. Vianna P, Santana S, Mckelvev W. Periodontal health and oral mucosal lesions as related to occupational exposure to acid mists. Community Dent Oral Epidemiol 2005; 33(5):341-8.

9. Instituto Nacional de Câncer. Ministério da Saúde. Ações de prevenção primária e secundária no controle do câncer. In: Ações de enfermagem para o controle do câncer: uma proposta de integração ensino-serviço. 3. ed. Rio de Janeiro: INCA; 2008.

10. Torres-Pereira CC, Angelim-Dias A, Melo NS, Lemos Jr CA, Oliveira EMF. Abordagem do câncer da boca: uma estratégia para os níveis primário e secundário de atenção em saúde. Cad Saude Publica 2012; 28:s30-s39.

11. Scully C, Kirby J. Statement on mouth cancer diagnosis and prevention. Br Dent J 2014; 216(1):37-8.

12. Lacerda JT, Simionato EM, Peres KG, Peres MA, Traebert J, Marcenes W. Dor de origem dental como motivo de consulta odontológica em uma população adulta. Rev Saude Publica 2004; 38(3):453-8.

13. Souza AMPA. A influência das condições de trabalho na atividade de cárie dental de trabalhadores em padaria e confeitaria: estudo de caso [Dissertação de mestrado]. Florianópolis: Programa de Pós-Graduação em Engenharia de Produção, Universidade Federal de Santa Catarina; 2002.

14. Brasil. Ministério da saúde. Relatório projeto Saúde Bucal Brasil 2010: pesquisa nacional de saúde bucal - resultados principais. Brasília, DF: 2011.

15. Almeida TF, Cangussu MCT, Chaves SCL, Amorim TM. Condições de saúde bucal em crianças, adolescentes e adultos cadastrados em unidades de saúde da família do município de Salvador, estado da Bahia, Brasil, em 2005. Epidemiol Serv Saúde 2012; 21(1):109-18.

16. Maia EG, Cavalcante GMS, Granville-Garcia AF, Lucas RSCC, Cavalcanti AL, D’Avila S. Condição de saúde bucal em trabalhadores atendidos no núcleo de saúde ocupacional de Campina Grande-PB. Arq Odontol 2012; 48(1):32-9.
17. Odontosites. Próteses dentárias mal adaptadas podem causar lesões na mucosa bucal. 2011. [citado 2012 dez]. Disponível em URL: http://www.odontosites.com.br/odonto/proteses-dentarias-mal-adaptadas-podem-causar-lesoes-na-mucosa-bucal.html.

18. Silva MES, Torres LHN, Sousa MLR. Impacto da perda dentária na qualidade de vida. Cien Saude Colet 2010; 15(3):841-50.

19. Silva MES, Magalhães CS, Ferreira EF. Perda dentária e expectativa da reposição protética: estudo qualitativo. Cien Saude Colet 2010; 15(3):813-20.

20. Lima LS, Silva KMG, Mello MVFM, Santos MS, Araújo MS, Cangussu MCT. Condição de urgência odontológica e fatores associados em trabalhadores da construção civil: Bahia, 2008. Rev Odontol Unesp [periódico on-line] 2013 [citado 2014 dez. 8]; 42(1):48-53. Disponível em: url:http://www.scielo. bt/scielo.php?pid=S1807-25772013000100009\&script=sci_ arttex\&tlng $=$ pt.

21. Brasil. Ministério da saúde. Relatório projeto saúde bucal Brasil 2003: condições de saúde bucal da população brasileira 2002-2003: resultados principais. Brasília, DF, 2004.

22. Cascaes AM. Doença periodontal e a autoavaliação da saúde bucal em adultos brasileiros [Dissertação de Mestrado]. Florianópolis: Universidade Federal de Santa Catarina, Programa de Pós Graduação em Saúde Pública; 2008.

23. Viana MIP, Sanrana VS. Exposição ocupacional a névoas ácidas e alterações bucais: uma revisão. Cad Saude Publica $2001 ; 17(6): 1335-44$

24. Teles MP. Condições de saúde bucal em trabalhadores de uma indústria metalúrgica da região metropolitana de Salvador, 2005 [Dissertação de Mestrado]. Salvador: Universidade Federal da Bahia, Faculdade de Odontologia; 2005.

25. Nogueira DP. Odontologia e saúde ocupacional. Rev Saude Publica 1972; (6):211-23.

26. Vianna MIP, Santana VS, Micklvey W. Periodontal health and oral mucosal lesions as related to occupational exposure to acid mists. Community Dent Oral Epidemiol 2005; 33:341-8.

27. Lucena EES, Costa DCB, Silveira EJD, Lima KC. Adoption of photoprotection measures on lip and perioral regions among beach workers in North Brazil. Int J Dermatol 2014; 53:480-5.

28. Pizzato E. A saúde bucal no contexto da saúde do trabalhador: análise dos modelos de atenção [Dissertação de Mestrado] Araçatuba: Universidade Estadual Paulista, Faculdade de Odontologia; 2002.

29. Conway DI, Petticrew M, Marlborough H, Berthiller J, Hashibe M, Macpherson LM. Significant oral câncer risk associated with low socioeconomic status. Evid Based Dent $2009 ; 10: 4-5$

30. Lucena EES, Costa DCB, Silveira EJD, Lima KC. Prevalence and factors associated to actinic cheilitis in beach workers. Oral Dis 2012; 18:575-9.

\section{Endereço para correspondência:}

Tatiana Frederico de Almeida

Rua João Bião de Cerqueira 251, Pituba

41830-580 Salvador, Bahia, Brasil

Telefone: 7133514447 / 99334886

E-mail: tatifrederico@yahoo.com.br

Recebido: 31/03/15. Aceito: 10/09/15. 